\section{A Biopsy-Negative \\ Esophageal Cancer: Diagnosis \\ by Combination of Bite Biopsy \\ and Endoscopic Mucosal \\ Resection Using a Cap-Fitted \\ Panendoscope (EMRC)}

Esophageal cancers which are not exposed to the lumen and which infiltrate deeply are difficult to diagnose using conventional bite biopsy, because such lesions are often covered by normal mucosa. We discovered that an accurate diagnosis could be made by a combination of endoscopic mucosal resection using a cap-fitted panendoscope (EMRC) $[1,2]$ and bite biopsy from the bottom of the artificial ulcer.

A 72-year-old man was admitted to Shinshu University Hospital complaining of regurgitation after ingesting solids and liquids. A barium esophagogram showed a tapered, narrowed, funnel-shaped lesion $4 \mathrm{~cm}$ in length in the middle of the esophagus. The surface of this structure was smooth. Esophagoscopy was carried out, but the endoscope could not be passed because the stenosis was very tight and firm. Mucosa in the stenotic region was smooth without ulceration (Figure 1) and iodine dye stained the area completely, leaving no unstained portion. Despite repeated conventional multiple biopsies and brushings for cytology, no pathological findings indicating esophageal cancer were obtained. To obtain biopsy specimens from the deeper region, a combination of EMRC and bite biopsy was performed. After EMRC at the oral site of the narrowed lesion, a bite biopsy was performed from the bottom of the artificial ulcer made by EMRC (Figure 2). The pathological findings regarding the resected mucosa obtained by EMRC revealed neither dysplastic nor malignant cells; however, the bite biopsy specimens showed squamous cell carcinoma. Finally we arrived at a diagnosis of diffusely infiltrative squamous cell carcinoma of the esophagus [3].

The combination of EMRC and bite biopsy is a useful diagnostic method for obtaining suitable biopsy specimens when tumor cells are present beneath the normal mucosa, as seen in the present patient.

\section{T. Yokoyama ${ }^{1}$, N. Nakamura ${ }^{2}$,}

K. Kiyosawa ${ }^{1}$, T. Akamatsu ${ }^{2}$

${ }^{1}$ 2nd Dept. of Internal Medicine,

Shinshu University School of Medicine,

Matsumoto, Japan

${ }^{2}$ Dept. of Endoscopy,

Shinshu University Hospital,

Matsumoto, Japan

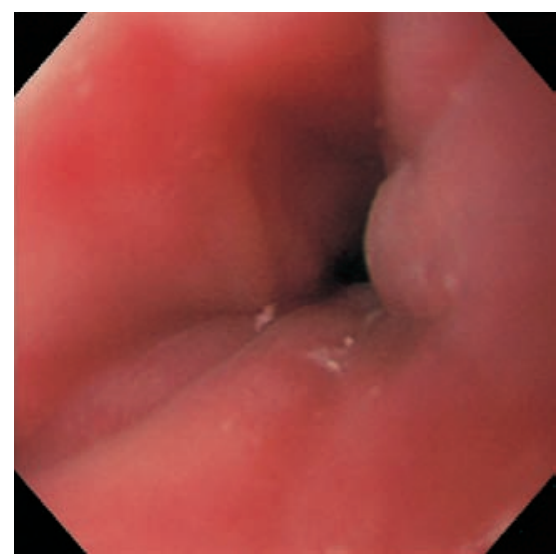

Figure 1 Endoscopic view reveals the oral side of the narrowing covered by normal esophageal mucosa

\section{References}

${ }^{1}$ Inoue H, Takeshita K, Hori H, et al. Endoscopic mucosal resection with a capfitted panendoscope for esophagus, stomach, and colon mucosal lesions. Gastrointest Endosc 1993; 39: 58-62

${ }^{2}$ Inoue H, Tani M, Nagai K, et al. Treatment of esophageal and gastric tumors. Endoscopy 1999; 31: 47-55

${ }^{3}$ Natsugoe S, Matsushita Y, Kijima F, et al. Diffusely infiltrative squamous cell carcinoma of the esophagus. Surg Today 1998; 28: 129-134

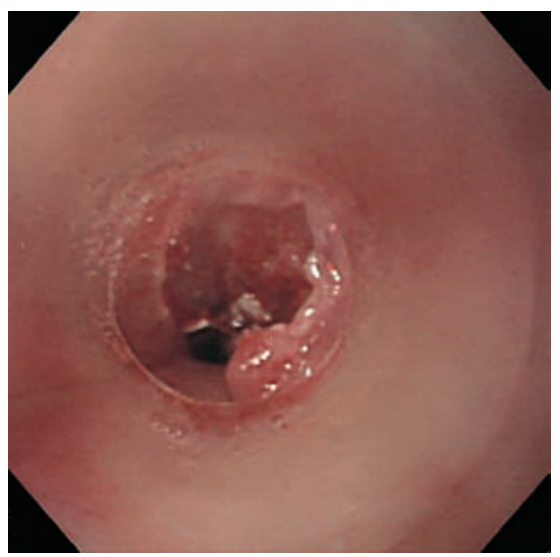

Figure 2 The surface of the normal mucosa is resected by endoscopic mucosal resection using a cap-fitted panendoscope (EMRC), and artificial ulceration is observed

\section{Corresponding Author}

T. Akamatsu, M.D.

Dept. of Endoscopy

Shinshu University Hospital

3-1-1 Asahi, Matsumoto

Nagano, 390-8621

Japan

Fax: +81-263-329412

E-mail: ta07260@hsp.md.shinshu-u.ac.jp 